\title{
A influência das quedas na qualidade de vida de idosos
}

\author{
The influence of falls on the quality of life of the aged
}

Adalgisa Peixoto Ribeiro ${ }^{1}$

Edinilsa Ramos de Souza ${ }^{1}$

Soraya Atie ${ }^{1}$

Amaro Crispim deSouza ${ }^{1}$

Arthur Orlando Schilithz ${ }^{1}$

${ }^{1}$ Centro Latino Americano deEstudos de Violênciae Saúde- CLAVES, Fundação Oswaldo Cruz. Av. Brasil, 4036/700, M anguinhos. 21040-361 Rio de Janeiro RJ. adalpeixoto@yahoo.com.br
Abstract The objective of this study is to analyze the effect of falls and their consequences on the quality of life of elderly people living in a lowincome community in the city of Rio de Janeiro. This article is part of an explorative research conducted at CLAVES, using quantitative and qualitative methods. In this study we used quantitative data about falls and quality of life. 72 elderly aged over 60 years participated in thestudy. $51,4 \%$ of them were females, $20,8 \%$ lived alone and $37,5 \%$ admitted having suffered a fall during the last year. Among the most frequently mentioned consequences were fractures $(24,3 \%)$, the fear of falling $(88,5 \%)$, abandonment of activities $(26,9 \%)$, change of habits $(23,1 \%)$ and immobilization (19\%). The analysis showed that falls have influence upon in the quality of life of the aged. TheW HOQ OL-Bref domain scores showed a reduction in the means of the group that had suffered falls during the last year in comparison to those who didn't fall, and the difference was moresignificant in thepsychological field. In short, falls arefrequent amongtheelderly and bring consequences that change the quality of life of these people in a negative way. Their incidence can be avoided by identifying the causes and developing appropriate preventive measures.

Key words Aging health, Falls, Quality of life
Resumo 0 objetivo desse artigo éanalisar o efeito das quedas e suas conseqüências na qualidade de vida de idosos de uma comunidade de baixa renda do município do Rio de Janeiro. Esteartigo é parte de um estudo exploratório realizado no CLAVES, que usou metodologia quantitativa e qualitativa. No presente trabalho, utilizou-se um recorte dos dados quantitativos referentes às que das eà qualidade devida. Participaram do estudo 72 idosos com idade de 60 ou mais anos, entre os quais $51,4 \%$ eram do sexo feminino, $20,8 \%$ moravam sozinhos e $37,5 \%$ admitiram ter caído no último ano. Entre as conseqüências mais citadas das quedas estão as fraturas (24,3\%), o medo de cair $(88,5 \%), 0$ abandono de atividades $(26,9 \%)$, a modificação de hábitos $(23,1 \%)$ e a imobilização (19\%). A análise mostrou que há influência das quedas na qualidade de vida dos idosos estudados. Em todos os domínios do WH OQ O L-Bref, houve redução nas médias do grupo que caiu no último ano em relação aos que não caíram e a diferença foi mais significativa no domínio psicológico. Enfim, as quedas são freqüentes entre os idosos e trazem conseqüências que alteram negativamentea qualidade de vida dessas pessoas. Sua ocorrência pode ser evitada com medidas preventivas adequadas, identificando causas e desenvolvendo métodos para reduzir sua ocorrência.

Palavras-chave Saúde do idoso, Quedas, Q ualidade devida 
Introdução

0 processo de envel hecimento vem acompanhado por problemas de saúde físicos e mentais provocados, freqüentemente, por doenças crônicas e quedas.

Desse modo, o conceito de saúde para este grupo populacional não pode se basear no parâmetro de completo bem-estar físico, psíquico e social preconizado pela O rganização M undial de Saúde, mas deve se reger pelo paradigma da capacidadefuncional ${ }^{1}$.

A quedaéum evento acidental quetem como resultado a mudança de posição do indivíduo para um nível mais baixo, em relação a sua posição inicial ${ }^{2}$, com incapacidade de correção em tempo hábil ${ }^{3}$ e apoio no solo. Não se considera queda quando o indivíduo somente cai de costas em um assento, por exemplo ${ }^{4}$.

A estabilidade do corpo depende da recepção adequada de informações através de componentes sensoriais, cognitivos, do sistema nervoso central e musculoesquelético de forma integrada. 0 efeito cumulativo de alterações relacionadas à idade, doenças e meio-ambiente inadequado parece predispor à queda 5 .

A origem da queda pode ser associada a fatores intrínsecos - decorrentes de alterações fisiológicas do envelhecimento, doenças e efeitos de medicamentos, e a fatores extrínsecos - circunstâncias sociais e ambientais que oferecem desafios ao idoso $0^{6}$.

As quedas apresentam diversos impactos na vida de um idoso, que podem incluir morbidade importante, mortalidade, deterioração funcional, hospitalização, institucionalização e consumo de serviços sociais e de saúde?. Além das conseqüências diretas da queda, os idosos restringem suas atividades devido a dores, incapacidades, medo de cair, atitudes protetoras de familiares e cuidadores ou atémesmo por aconsel hamento de profissionais de saúde ${ }^{8,5}$.

Em 2005, ocorreram 61.368 hospitalizações por queda de pessoas com 60 anos ou mais de idade, de ambos os sexos, representando 2,8\% detodas as internações de idosos no paíse $54,4 \%$ das internações por todas as lesões e envenenamentos neste grupo etário. No estado do Rio de Janeiro, o percentual de hospitalizações por queda entre os idosos atingiu $3,7 \%$ de todas as internações de idosos no estado ${ }^{9}$. Na capital deste estado, foram 1.934 hospitalizações de idosos devido às quedas, expressando $6,5 \%$ das internações por todas as causas das pessoas nesta faixa etária e $60,5 \%$ de todas as lesões e envenenamen- tos que geraram hospitalizações entre este grupo. É preciso levar em consideração que essas foram as quedas mais graves e que exigiram cuidados hospitalares mais intensos, mas umagrande parcela desses eventos não leva o idoso a procurar os serviços de saúde por não apresentarem seqüelas tão graves como uma fratura ou algum outro tipo de ferimento. Além disso, alguns casos são atendidos em serviços de emergência ou postos de saúde.

Além da morbidade advinda da queda, a mortalidade entre os idosos por esta causa externa é muito comum e seus registros podem ser encontrados em diversos estudos já realizados no país. Fabrício et al. ${ }^{6}$ mostraram em seu trabaIho, realizado com cinqüenta idosos, que $28 \%$ deles faleceram por conseqüências diretas da queda, entre elas, fraturas e lesões neurológicas. Outro fato a ser levado em consideração é que a maioria dos óbitos ocorreu entre as mulheres (78,5\%). Somente no estado do Rio de Janeiro, 441 pessoas idosas morreram por causa das quedas em 2004 e essas mortes representaram 1,0\% de todos os óbitos de idosos no estado. No Brasil, em 2004, as mortes por queda entre os idosos chegaram a 3.024 e esse percentual representou 0,6\% de todas as mortes de idosos no país 9 .

Entreosidosos, écomum acontecerem multimorbidades ereincidência das quedas, gerando incapacidades parciais ou dependência e pior qualidade de vida. A qualidade de vida da pessoa idosa se vê ainda mais deteriorada quando estão presentes problemas de saúde, muito freqüentes na idade avançada, a violência e certos fatores de risco para quedas e outros acidentes.

O conceito de qualidade de vida podeser concebido como uma representação social com parâmetros objetivos - satisfação das necessidades básicas e criadas pelo grau de desenvolvimento econômico e social da sociedade - e subjetivos bem-estar, felicidade, amor, prazer, realização pessoal ${ }^{10}$. Além desses parâmetros, o conceito também inclui critérios de satisfação individual e debem-estar coletivo. Ao analisar a qualidade de vida, devem-se considerar fatores políticos e de desenvolvimento humano. 0 grau de satisfação humana nas diferentes esferas de vida (familiar, amorosa, ambiental, social, profissional e existencial) relaciona-se ao padrão de conforto e bem-estar estabelecido pela sociedade, historicamente. Em relação à saúde, "as noções se unem em uma resultante social da construção coletiva dos padrões de conforto e tolerância que determinada sociedade estabelece, como parâmetros, para si" ${ }^{10}$. Segundo $M$ atos $^{11}$, quanto mais apri- 
morada éa democracia, mais ampla éa noção de qualidade de vida.

Vecchia et al. ${ }^{12}$, em uma pesquisa sobrea compreensão do que vem a ser qualidade de vida para os idosos, encontraram diversos fatores que influenciam a vida no sentido de uma boa qualidade. São eles: relacionamentos interpessoais, boa saúde física e mental, bens materiais (casa, carro, salário, acesso a serviços de saúde), lazer, trabaIho, espiritualidade, honestidade e solidariedade, educação (ao longo da vida) e ambiente favorável (sem poluição esem violência). Essesfatorestambém são confirmados por outros autores ${ }^{13-15}$.

Embora não haja um consenso acerca da de finição de qualidade devida, a O rganização Mundial de Saúde ${ }^{16}$ considera que a subjetividade, a muldimensionalidade e as dimensões positivase negativas são aspectos fundamentais para a compreensão deste constructo.

0 desenvolvimento desses aspectos levou à definição de qualidade de vida como "a percepção do indivíduo de sua posição na vida no contexto da cultura e sistema devalores nos quais ele viveeem relação aos seus objetivos, expectativas, padrões e preocupações" ${ }^{\prime 17}$.

0 objetivo do presente estudo é analisar o efeito das quedas e suas conseqüências na qualidade de vida de idosos moradores de uma comunidade de baixa renda do município do Rio deJaneiro.

\section{Metodologia}

Este artigo é parte de um estudo exploratório realizado no Centro Latino Americano de Estudos sobre Violência e Saude/CLAVES ${ }^{18}$, no município do Rio de Janeiro, que usou metodologia quantitativa e qualitativa. No presente trabalho, utilizou-se um recorte dos dados quantitativos referentes às quedas e à qualidade de vida.

Foram incluídos 72 idosos com idade de 60 ou mais anos, em uma amostra de conveniência, moradores de uma comunidade de baixa renda do município do Rio de Janeiro. Esta comunidade se localiza na zona norte da cidade, nas proximidades da Fundação O swaldo Cruz, e é composta por cerca de 50.000 moradores. Possui cerca de 12.000 domicílios, sendo que $30 \%$ deles são construídos em áreas consideradas irregulares, provisórias ou de risco ${ }^{19}$. Concentra grandes e graves insuficiências, precariedades eausência de infra-estrutura urbana e de serviços públicos de educação, saúde, cultura, lazer e segurança pública. Essas áreas têm uma vocação para 0 de- senvolvimento de organizações socioculturais enraizadas na vida local. N essas organizações, existem autoridades informais locais, legitimadas por identidades coletivas territoriais e que podem ser de natureza legal ou ilegal, como é o caso do narcotráfico, que negocia até mesmo 0 acesso da favela aos bens públicos da cidade ${ }^{20}$. A violência perpetrada pelo grupo de narcotraficantes que controla o comércio de drogas ilícitas no local éuma situação constante vivenciada pelos moradores dessa comunidade.

Para a coleta de informações junto aos idosos, foi elaborado um questionário com questões fechadas e abertas sobre a situação sociodemográfica, quedas e qualidade de vida. Neste questionário, foi incluída uma escala padronizada de Avaliação da Qualidade de Vida da Organização Mundial de Saúde - WHOQOL em sua versão abreviada.

A versão abreviada do WHOQOL se constitui por 26 questões subdivididas em quatro domínios de análise. São duas questões gerais de qualidade de vida e 24 referentes aos domínios físico, relativo a níveis de dor física e tratamento médico; psicológico, referentea aceitação da aparência física, como aproveita a vida, etc; relações sociais com parentes, amigos, conhecidos e, finalmente, meio ambiente, que investiga quanto 0 ambiente é saudável para 0 idoso ${ }^{16}$.

As informações coletadas foram codificadas, digitadas e processadas em microcomputador. Para a entrada de dados, foi elaborada uma máscara utilizando o software M icrosoft Access. Uma análise crítica dos dados foi feita para categorizar as questões abertas e corrigir erros originados nas fases de aplicação, codificação e digitação. Posteriormente, os dados foram exportados e manuseados para os softwares Microsoft Excel e SPSS (Statistical Package for the Social Sciences), versão 13.0, em que se realizou a descrição de freqüências simples, análises uni e bivariadas de variáveis e alguns testes estatísticos.

0 nível de significância estabelecido a priori foi de $5 \%$. Entretanto, o simplesfato de estabelecer este ponto de corte não invalidou a hipótese de diferença nos casos em que a probabilidade de encontrar a hipótese nula ao acaso fosse próximo a 0,05 .

Foram realizadas comparações entre médias por domínio para a escala de Avaliação de Qualidade deVida da Organização M undial deSaúde (WHOQOL-Bref) entre os grupos de idosos positivos ou não para quedas e das distintas faixas etárias (de 60 a 69 anos; 70 a 79 anos; 80 a 91 anos). Foi utilizado o teste t de Student com a 
finalidade de encontrar diferenças estatisticamente significativas entre os grupos segundo cada domínio. 0 testet de Student éuma técnica empregada quando desejamos comparar duas mé dias amostrais para verificar se elas são estatisticamente diferentes. Esse teste tem o pressuposto de que as distribuições das variáveis contínuas utilizadas sejam aproximadamente normais (gaussianas). Tal pressuposto foi verificado e confirmado para cada distribuição dos domínios da escala por grupo de idosos (que apresentaram quedas ou não).

Todos assinaram um termo de consentimento para participar da pesquisa que foi aprovada pelo ComitêdeÉtica e Pesquisa da Escola Nacional deSaúdePública/Fiocruz.

\section{Resultados ediscussão}

Dos 72 idosos do presente estudo, 51,4\% eram do sexo feminino e $20,8 \%$ moravam sozinhos. A faixa etária de 60 a 69 anos compreendia 41,7\% dos sujeitos, de 70 a 79 anos, outros $41,7 \%$ e a faixa de 80 a 91 anos de $16,6 \%$ dos idosos.

Entreeles, 37,5\% admitiram ter caído no último ano e, dos que caíram, 70,4\% referiram uma só queda, enquanto $29,6 \%$ relataram mais de uma queda. Essa proporção de quedas é próxima à encontrada em um grupo de mulheres com 60 ou mais anos que freqüentam um programa de atividade física da Universidade do Estado do Rio de Janeiro, no qual $43,0 \%$ delas sofreram quedas no último ano e bem menor que a proporção encontrada por Fabrício et al. ${ }^{6} \mathrm{em}$ um grupo de idosos em Ribeirão Preto, onde 54,0\% deles haviam caído no último ano.

O local onde ocorre a queda parece estar re lacionado com as habilidades que o idoso apresenta para realizar as tarefas da vida diária e com a idade. Para M asud e M orris 7 , pessoas com idade menor que 75 anos têm maior probabilidade de cair em ambientes externos e os idosos com mais de 75 anos caem mais no interior de suas próprias residências. A maioria das quedas detectadas no presente estudo ocorreu na residência do idoso (59,5\%), mas deve-se considerar os $40,5 \%$ dos casos ocorridos fora da residência. N os idosos do trabal ho de Ribeirão Preto ${ }^{6}, 66,0 \%$ das quedas ocorreram dentro da residência do idoso e $22,0 \%$, na rua.

Como conseqüência da queda, $24,3 \%$ dos idosos informaram ter sofrido fraturas, a mais freqüente foi a de fêmur (33,3\%) e al guns fraturaram mais de um membro. As quedas levaram à necessidade de atendimento médico em 48,6\% dos casos. Desses, 58,3\% recorreram a um hospital de traumatologia, $77,8 \%$ a serviço de pronto socorro ou emergência, $16,7 \%$ a postos de saúde e para $5,6 \%$ não se obteve essa informação. A freqüente ocorrência de fraturas em conseqüência das quedas é apontada também na literatura nacional e internacional, sobretudo as fraturas do fêmur ${ }^{3}$.

Além de fraturas, as quedas provocam uma série de outras conseqüências, conforme se pode ver no Gráfico 1. O medo de voltar a cair passou a fazer parte da vida do idoso e foi referido por $88,5 \%$ dos 26 idosos que afirmaram haver tido alguma conseqüência. Dentre essas, se destacaram 0 abandono de certas atividades (26,9\%), a modificação de hábitos (23,1\%) ea imobilização (19\%). Em relação aos problemas de saúde causados por quedas ou por outras condições, os dados da Pesquisa Nacional por Amostragem de Domicílios/PNAD de 1998 revelam que $13,9 \%$ dos idosos brasileiros deixaram de realizar alguma atividade habitual nas duas últimas semanas por motivo de saúde e este relato aumentou com a idade de forma consistente em ambos os sexos ${ }^{21}$.

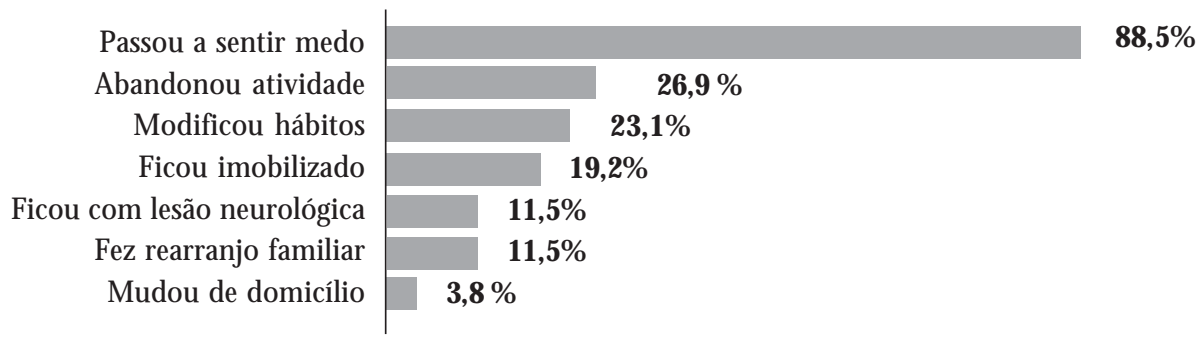

Gráfico 1. Distribuição proporcional dos idosos segundo conseqüência da queda. Comunidade do Rio de Janeiro, 2005. 
Idosos de Ribeirão Preto ${ }^{6}$ apresentaram como principais conseqüências das quedas as fraturas (64\%), o medo de cair (44\%), o abandono de atividades ( $12 \%$ ) e a modificação de hábitos (18\%), imobilização (4\%), rearranjo familiar $(2 \%)$, lesão neurológica (6\%) e mudança de domićlío (10\%).

0 medo de cair, que entre os idosos do presente estudo foi encontrado em alta proporção, é uma conseqüência destacada por vários autores etraz consigo alterações como perda de autonomia e independência para as atividades de vida diária, diminuição das atividades sociais e sentimentos de fragilidade e insegurança $a^{4,6}$.

Por outro lado, o medo pode atuar também como um fator protetor na medida em que 0 idoso, em função dele, adota comportamentos preventivos. Isto aparece de certa forma nos dados do Rio de Janeiro comparados aos de Ribeirão Preto. Entre os idosos da primeira cidade, embora tenha havido menos fraturas devido a quedas, elas parecem ter sido mais graves, pois redundaram em mais lesão neurológica eimobilizações. Talvez por isso, o sentimento de medo esteve bem mais presente nos idosos cariocas, juntamente com os demais comportamentos investigados - abandonar certas atividades, modificar hábitos, buscar rearranjos familiares.

Entre os fatores de risco intrínsecos investigados, destacam-se os problemas de saúde relatados por $77,8 \%$ dos idosos. Entre eles, os cardiovasculares $(67,8 \%)$, osteomusculares $(42,8 \%)$ eendócrinos (28,6\%). Q uando indagados se possuíam algum problema psiquiátrico, todos responderam negativamente. Vale destacar que esta questão foi feita deforma genérica e a interpreta- ção ficou a critério dos idosos, nenhuma opção de doença psiquiátrica foi oferecida como opção de resposta. Dificuldades visuais $(58,3 \%)$ e de locomoção (38,9\%), além de problemas nos pés $(47,1 \%)$ foram amplamente referidos.

Algumas alterações psíquicas que não necessariamentese traduzem em doenças psiquiátricas foram aqui denominadas problemas psicológicos e questionados enquanto fator intrínseco de risco para queda. Entre os mais citados, encontram-se a dificuldade de sono e o sentimento de tristeza, como se pode ver no Gráfico 2.

A dificuldade desono esentimento detristeza podem estar associados a sintomas depressivos; 0 aumento da freqüência urinária, perda de apetite, pal pitações e agitação podem denotar ansiedade, mas também reações adversas pelo uso de medicamentos ou sintomas de alguma outra doença, como as cardiovasculares, por exemplo. O s eventos psicossociais negativos e o estresse são fatores que podem levar os idosos a alterações emocionais e a vulnerabilidade a quedas ${ }^{22}$.

0 uso de medicamentos, um outro fator intrínseco muito freqüente entre idosos, foi relatado no presente estudo por $69,4 \%$ deles. Destacam os anti-hipertensivos $(45,7 \%)$ e os antiinflamatórios (25,0\%). 0 uso de quatro ou mais medicamentos de forma concomitante (polifarmácia) ocorre entre $20 \%$ dos idosos. Essa proporção de polifarmácia é bem menor se comparada com a encontrada entre os idosos de Ribeirão Preto $(42,0 \%)^{6}$. Segundo Nascimento ${ }^{23}$, o idoso é alvo de uma medicação indiscriminada, abusi va eàs vezes desnecessária, o que causa efeitos adversos pelo mecanismo de interação medicamentosa que pode levar até à morte.

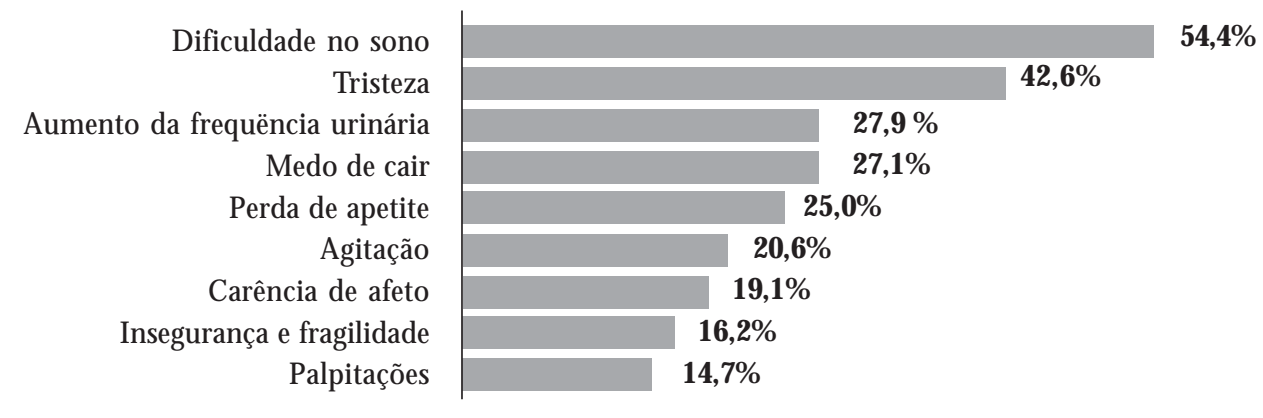

Gráfico 2. Distribuição proporcional dos idosos segundo problemas psicológicos no último ano.

Comunidade do Rio de Janeiro, 2005 
Além dos fatores intrínsecos, as condições ambientais também influenciam o risco de cair. Os dados do presente estudo chamam a atenção para a precariedade das moradias improvisadas da área de residência dos idosos, que não oferece as condições necessárias para sua segurança.

Como fatores extrínsecos, foram aqui consideradas algumas condições ambientais com as quais os idosos têm que lidar em sua casa, como se podever no Gráfico 3. Entre os fatores derisco para acidentes mais citados, encontram-seo piso escorregadio na casa ( $70,6 \%)$, piso escorregadio no banheiro $(66,2 \%)$, calçados inadequados $(64,7 \%)$ e degraus na soleira da porta $(55,9 \%)$. Além desses, várias outras situações de risco para quedas e outros acidentes foram relatadas como fazendo parte do ambiente doméstico e do cotidiano dos idosos que responderam ao questionário. Estes dados chamam a atenção para a precariedade das moradias improvisadas da área, que não oferecem as condições necessárias para a segurança dos idosos.

Diante das experiências de quedas, os fatores de risco e as condições de saúde dos idosos pesquisados, buscou-se, a partir do instrumento padronizado pela Organização Mundial de Saúde, verificar a qualidade de vida desse grupo. Os dados da Tabela 1 mostram os resultados das análises dos domínios do WHOQOL-Bref, segundo presença ou ausência de quedas no último ano.

A análise mostrou que háinfluência das que das na qualidade de vida dos idosos estudados.
Em todos os domínios da escala aplicada, houve uma redução nas médias do grupo que caiu no último ano. Essa diferença foi mais significativa no domínio psicológico.

Uma possível explicação para esse achado seria um aumento dedependência dosidosos como conseqüência da queda, após as quais eles passariam a experimentar sentimentos negativos, alterações na memória e concentração, baixa auto-estima e alterações na imagem corporal e aparência.

Esses resultados também foram apontados por $\mathrm{H}$ wang et al. ${ }^{15}$, que estudaram idosos de Taiwan e encontraram menores médias no grupo que apresentou quedas no último ano.

A qualidade devida depende defatoresintrínsecos - condições de saúde e atitudes frente aos acontecimentos da vida - eextrínsecos - recursos financeiros e ambientais que rodeiam os indivíduos. Para as pessoas que vivem em comunidades de baixa renda, como é o caso desses idosos, todas as dificuldades inerentes ao ambiente que os cerca - precárias condições de moradia, infraestrutura deficiente e situações de violência - influenciam a percepção da qualidade de vida.

Um ambiente propício e satisfatório para o idoso é aquele que oferece segurança, é funcional, proporciona estímulo e controle pessoal, facilita a interação social, favorece a adaptação às mudanças e é familiar para o idoso ${ }^{24}$. Como era de se esperar, o domínio de meio ambiente apresentou a pior média no conjunto dos dados ( Tabela 1), mostrando as condições adversas de vida existentes em áreas especiais como a estudada.

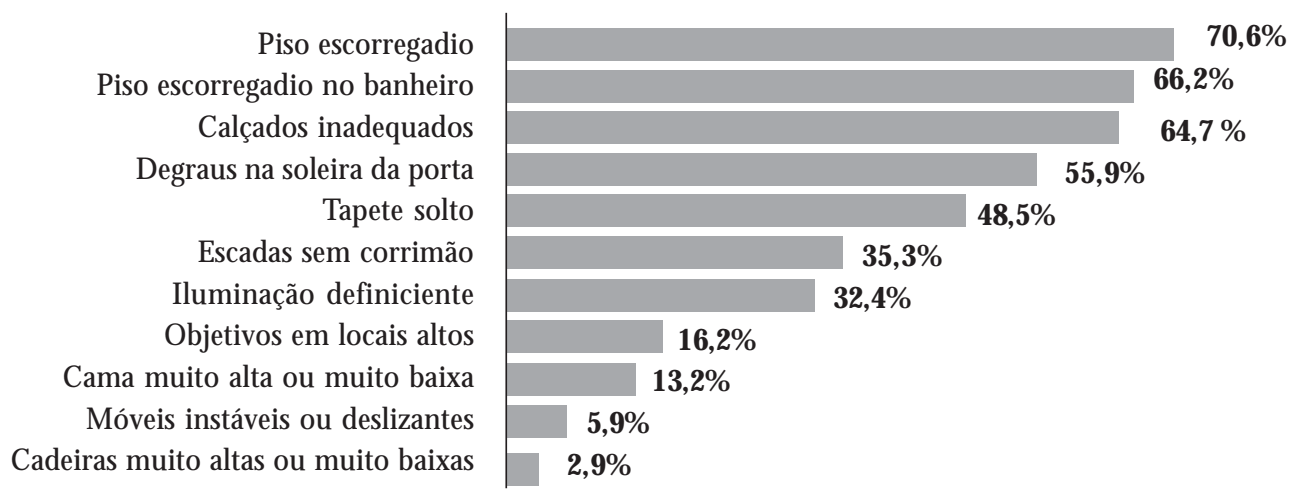

Gráfico 3. Distribuição proporcional dos idosos segundo fatores de riscos ambientais presentes em sua residência. Comunidade do Rio de Janeiro, 2005. 
0 meio ambiente é fortemente associado à qualidade de vida entre os idosos devido à sua relação com a prevenção de quedas, a interação social, o envolvimento em atividades do cotidiano, a independência, segurança e proteção e 0 bem-estar psicológico ${ }^{15}$.

Em relação ao grupo de idosos como um todo, as médias apresentadas são melhores que as médias dos idosos estudados por $\mathrm{H}$ wang et al. ${ }^{15}$. Em uma estratificação por presença de comorbidades crônicas, depressão, enfraquecimento cognitivo e quedas, os idosos tai waneses obtiveram médias menores se comparadas às médias dos idosos cariocas nos domíniosfísico, psicológico e de relações sociais. Para o domínio do meio ambiente, os cariocas apresentam médias menores que os idosos de Tai wan, demonstrando a influência negativa do ambienteda favela na qualidade de vida deles (Tabela 2).
Comparando com as médias dos idosos com depressão, pacientes deum hospital psiquiátrico da Austrália ${ }^{25}$, os idosos do presente estudo apresentaram médias maiores nos domínios físico e psicológico e estão em desvantagem em relação aos idosos com depressão nos domínios de relações sociais e meio ambiente.

Não parecem existir diferenças em relação às faixas etárias quando se observa cada domínio separadamente, embora ocorra um crescimento da média em função do aumento da idade em relação às duas questões gerais sobre qualidade de vida e satisfação com a saúde. Esse aumento é maior no grupo das mulheres que na faixa etária de 60 a 69 anos, obteve média 13,0, na faixa de 70 a 79 anos, 14,73, de 80 a 91 anos, a média foi 16,00. Uma hipótese para esse achado inusitado seria que as mulheres têm a percepção de que sua vida melhora quando estão mais velhas, pois já

Tabela 1. Distribuição das médias dos domínios da qualidade de vida dos idosos segundo experiência de quedas. Comunidade do Rio de Janeiro, 2005.

\begin{tabular}{|c|c|c|c|c|c|}
\hline Queda & $\begin{array}{l}\text { Domínio } \\
\text { físico }\end{array}$ & $\begin{array}{l}\text { Domínio } \\
\text { psicológico }\end{array}$ & $\begin{array}{l}\text { Domínio } \\
\text { relações sociais }\end{array}$ & $\begin{array}{l}\text { Domínio meio } \\
\text { ambiente }\end{array}$ & $\begin{array}{l}\text { Qualidade de vida + } \\
\text { satisfação com saúde }\end{array}$ \\
\hline \multicolumn{6}{|l|}{ Presença } \\
\hline M édia & 13,04 & 13,85 & 13,63 & 11,56 & 13,60 \\
\hline D.P. & 2,40 & 2,04 & 2,57 & 1,98 & 3,08 \\
\hline Alpha & 0,746 & 0,618 & 0,739 & 0,779 & 0,712 \\
\hline \multicolumn{6}{|l|}{ Ausência } \\
\hline M édia & 13,64 & 14,62 & 14,31 & 11,80 & 14,28 \\
\hline D.P. & 1,92 & 1,41 & 2,25 & 1,18 & 1,52 \\
\hline Alpha & 0,674 & 0,476 & 0,638 & 0,343 & 0,372 \\
\hline \multicolumn{6}{|l|}{ Total } \\
\hline M édia & 13,41 & 14,33 & 14,05 & 11,71 & 14,03 \\
\hline D.P. & 2,01 & 1,63 & 2,15 & 1,26 & 2,21 \\
\hline Alpha & 0,709 & 0,572 & 0,686 & 0,614 & 0,604 \\
\hline
\end{tabular}

Tabela 2. Distribuição comparativa das médias dos domínios da qualidade de vida dos grupos de idosos.

\begin{tabular}{lcccc}
\hline \multicolumn{1}{c}{ Grupo } & $\begin{array}{c}\text { Domínio } \\
\text { físico }\end{array}$ & $\begin{array}{c}\text { Domínio } \\
\text { psicológico }\end{array}$ & $\begin{array}{c}\text { Domínio } \\
\text { relações sociais }\end{array}$ & $\begin{array}{c}\text { Domínio meio } \\
\text { ambiente }\end{array}$ \\
\hline $\begin{array}{l}\text { Comunidade do Rio de Janeiro, } 2005 \\
\text { M édia }\end{array}$ & 13,41 & 14,33 & 14,05 & 11,71 \\
$\begin{array}{l}\text { H wang et al. }{ }^{15} \\
\text { M édia }\end{array}$ & 13,00 & 13,00 & 13,00 & 13,00 \\
$\begin{array}{c}\text { Naumann e Byrne }{ }^{25} \\
\text { M édia }\end{array}$ & 12,45 & 11,63 & 15,12 & 14,17 \\
\hline
\end{tabular}


teriam perdido seus cônjuges e se sentem mais livres da submissão e dos cuidados dedicados a eles e aos filhos.

\section{Considerações finais}

As quedas na população idosa são freqüentes e determinam complicações que alteram negativamente a qualidade de vida dessas pessoas. Sua ocorrência pode ser evitada com medidas preventivas adequadas, identificando causas edesenvolvendo métodos para reduzir sua ocorrência.

Seguindo o que estabelece a Política Nacional de Saúde do Idoso, em relação à promoção do envelhecimento saudável com manutenção da pessoa idosa em seu ambiente familiar e com capacidade funcional preservada, um programa quetrabal hea conscientização da sociedadee dos próprios idosos sobre medidas que diminuam os riscos para a queda é imperioso e urgente.

Vale ressaltar que, em comunidades de baixa renda, como é o caso da área de moradia dos idosos deste estudo, a urbanização desses espaços públicos seria um importante passo para reduzir os riscos com que os idosos convivem em suas atividades diárias.

Com o acentuado crescimento de idosos na população brasileira e as dificuldades enfrentadas por eles em relação à saúde e às condições socioeconômicas, advoga-se aqui uma discussão de programas específicos que se voltem à prevenção das quedas e à promoção do envelhecimento saudável com qualidade de vida.

\section{Colaboradores}

A P Ribeiro eER Souza participaram da concepção, análise dos dados e escrita do artigo; S Atie e AC Souza colaboraram na escrita do artigo; AO Schilithz participou da análise estatística dos dados.

\section{Agradecimentos}

À Fundação do Amparo à Pesquisa do Estado do Rio de Janeiro pelo apoio fornecido ao estudo 0 Estado de direitoe a violência contra o idoso. 


\section{Referências}

1. Ramos M P. Apoio Social e saúde entre idosos. Sociologias 2002; 4(7):156-175.

2. Moura RN, Santos FC, Driemeier M, Santos LM, Ramos LR. Quedas em idosos: fatores de risco associados. Gerontol 1999; 7(2):15-21.

3. Santos M LC, Andrade M C. Incidência de quedas relacionada aos fatores de risco em idosos institucionalizados. Rev Baiana de Saúde Pública 2005; 29(1):57-68.

4. Paixão Júnior $C M$, H eckmann M. Distúrbios da postura, marcha e quedas. In: Freitas EV, Ligia Py, Flávio Aluizio Xavier Cançado, Milton Luiz Gorzoni, organizadores. Tratado de geriatria e gerontologia. Rio de Janeiro: Guanabara Koogan; 2003. p. 624-634.

5. Pereira SRM, Buksman S, Perracini M, Py L, Barre to $K M L$, Leite VMM. Quedas em idosos. Rio de Janeiro: Sociedade Brasileira de Geriatria e Gerontologia; 2001.

6. Fabrício SCC, Rodrigues RAP, Costa Junior M L. Causas e conseqüências de quedas de idosos atendidos em hospital público. Rev. Saúde Pública 2004; 38(1):93-99.

7. Masud T, Morris RO. Epidemiology of falls. Age Ageing 2001; 30(s4):3-7.

8. Downton J. Falls. In: Tallis RC, Fillit HM, Brockleurst JC, editors. Brocklehurst's textbook of geriatric medicine and gerontology. London: Churchill Livingstone; 1998. p. 1683.

9. Brasil. M inistério da Saúde. [acessado 2006 Jul 25]. Disponível em: www.datasus.gov.br

10. M inayo M CS, Hartz ZA, Buss PM. Qualidade de vida e saúde: um debate necessário. Cien Saude Colet 2000; 5(1):7-18.

11. Matos 0 . As formas modernas de atraso. Folha de São Paulo 199927 Set; p. 3

12. Vecchia RD, Ruiz T, Bocchi SCM, Corrente JE. Qualidade de vida na terceira idade: um conceito subjetivo. Rev Bras Epidemiol 2005; 8(3):246-252.

13. Koo J, Rie J, Park K. Age and gender differences in affect and subjective well-being. Geriatrics and Gerontology International 2004; 4:268-270.

14. Xavier FM F, Ferraz MPT, Maré N, Escosteguy NU, Moriguchi EH. Elderly people's definition of quality of life. Rev Bras Psiquiatr 2003; 25(1):31-39.
15. H wang HF, Liang WM, Chiu YN, Lin MR. Suitability of the WHOQOL-BREF for community-dwelling older people in Taiwan. Age Ageing 2003; 32:593-600.

16. Organização $M$ undial de Saúde. Divisão de Saúde M ental Grupo WHOQOL. Versão em português dos instrumentos de avaliação de qualidade de vida (WHO Q OL). Genebra: OM S; 1998.

17. WHOQOL Group. The development of the World $\mathrm{H}$ ealth Organization quality of life assessment instrument (the WHOQOL). In: Orley J, Kuyken W, editors. Quality of life assessment: international perspectives. Heidelberg: Springer Verlag; 1994. p. 41-60.

18. Souza ER, Ribeiro AP, Atie S, Souza A. O estado de direito e a violência contra o idoso. Rio de Janeiro: Claves/Ensp/Fiocruz; 2006.

19. Bodstein R, Zancan L, Estrada DD. Manguinhos: guia de equipamentos e iniciativas sociais. Rio de Janeiro: Fiocruz; 2001.

20. Burgos M B. Cidade, territórios e cidadania. Dados 2005; 48(1):189-222.

21. Lima-Costa MF, Barreto S, Giatti L, U choa E. Desigualdade social e saúde entre idosos brasileiros: um estudo baseado na Pesquisa Nacional por Amostra de Domicílios. Cad Saúde Pública 2003; 19(3):745-757.

22. M artins VMC. Quedas em pacientes geriátricos [monografia de especialização]. Rio de Janeiro (RJ): Escola Nacional de Saúde Pública/Fundação Oswaldo Cruz; 1999.

23. Nascimento JSF. Farmacologia e terapêutica na veIhice. In: Freitas EV, Py L, Cançado FAX, Gorzoni $M L$, organizadores. Tratado de geriatria e gerontologia. Rio de Janeiro: Guanabara Koogan; 2002.

24. Diogo MJD'E. Envelhecimento da pessoa portadora de necessidades especiais. In: Neri AL, coordenadora. Saúde e qualidade de vida na velhice. Coleção Velhice e sociedade. Campinas: Alínea; 2004.

25. Naumann VJ, Byrne GJA. WHOQOL-BREF as a measure of quality of life in older patients with depression. International Geriatrics 2004; 16(2):159-173.

Artigo apresentado em 04/09/2006

Aprovado em 25/09/2006

Versão final apresentada em 28/09/2006 